Journal for ImmunoTherapy of Cancer

\title{
Baseline BMI and BMI variation during first line pembrolizumab in NSCLC patients with a PD-L1 expression $\geq 50 \%$ : a multicenter study with external validation
}

Alessio Cortellini (D) ,1,2 Biagio Ricciuti, ${ }^{3,4}$ Marcello Tiseo, ${ }^{5,6}$ Emilio Bria, ${ }^{7,8}$ Giuseppe L Banna, ${ }^{9}$ Joachim GJV Aerts, ${ }^{10}$ Fausto Barbieri, ${ }^{11}$ Raffaele Giusti, ${ }^{12}$ Diego L Cortinovis, ${ }^{13}$ Maria R Migliorino, ${ }^{14}$ Annamaria Catino, ${ }^{15}$ Francesco Passiglia, ${ }^{16}$ Mariangela Torniai, ${ }^{17}$ Alessandro Morabito (D),${ }^{18}$ Carlo Genova, ${ }^{19}$ Francesca Mazzoni, ${ }^{20}$ Vincenzo Di Noia, ${ }^{21}$ Diego Signorelli, ${ }^{22}$ Alain Gelibter, ${ }^{23}$ Mario Alberto Occhipinti, ${ }^{23}$ Francesca Rastelli, ${ }^{24}$ Rita Chiari, ${ }^{25}$ Danilo Rocco, ${ }^{26}$ Alessandro Inno, ${ }^{27}$ Michele De Tursi, ${ }^{28}$ Pietro Di Marino, ${ }^{29}$ Giovanni Mansueto, ${ }^{30}$ Federica Zoratto, ${ }^{31}$ Francesco Grossi, ${ }^{32}$ Marco Filetti, ${ }^{12}$ Pamela Pizzutilo, ${ }^{15}$ Marco Russano, ${ }^{33}$ Fabrizio Citarella, ${ }^{33}$ Luca Cantini, ${ }^{10,17}$ Giada Targato, ${ }^{34}$ Olga Nigro, ${ }^{35}$ Miriam G Ferrara, ${ }^{7,8}$ Sebastiano Buti, ${ }^{5}$ Simona Scodes, ${ }^{36}$ Lorenza Landi, ${ }^{36}$ Giorgia Guaitoli, ${ }^{11}$ Luigi Della Gravara, ${ }^{26}$ Fabrizio Tabbò, ${ }^{16}$ Serena Ricciardi, ${ }^{14}$ Alessandro De Toma, ${ }^{22}$ Alex Friedlaender, ${ }^{37}$ Fausto Petrelli, ${ }^{38}$ Alfredo Addeo, ${ }^{37}$ Giampiero Porzio, ${ }^{2}$ Corrado Ficorella ${ }^{1,2}$

\section{ABSTRACT}

Tiseo M, et al. Baseline BMI and $\mathrm{BMI}$ variation during first line pembrolizumab in NSCLC patients with a PD-L1 expression $\geq 50 \%$ : a multicenter study with external validation. Journal for ImmunoTherapy of Cancer 2020;8:e01403. doi:10.1136/jitc-2020-001403

- Additional material is published online only. To view please visit the journal online (http://dx.doi.org/10.1136/jitc2020-001403).

Accepted 02 September 2020

Check for updates

(C) Author(s) (or their employer(s)) 2020. Re-use permitted under CC BY-NC. No commercial re-use. See rights and permissions. Published by BMJ.

For numbered affiliations see end of article.

Correspondence to Dr Alessio Cortellini; alessiocortellini@gmail.com expression.
Background The association between obesity and outcomes in patients receiving programmed death-1/ programmed death ligand-1 (PD-L1) checkpoint inhibitors has already been confirmed in pre-treated non-small cell lung cancer (NSCLC) patients, regardless of PD-L1 tumor

Methods We present the outcomes analysis according to baseline body mass index (BMI) and BMI variation in a large cohort of metastatic NSCLC patients with a PD-L1 expression $\geq 50 \%$, receiving first line pembrolizumab. We also evaluated a control cohort of metastatic NSCLC patients treated with first line platinum-based chemotherapy. Normal weight was set as control group. Results 962 patients and 426 patients were included in the pembrolizumab and chemotherapy cohorts, respectively. Obese patients had a significantly higher objective response rate (ORR) $(0 \mathrm{R}=1.61$ (95\% Cl: 1.042.50)) in the pembrolizumab cohort, while overweight patients had a significantly lower ORR (OR=0.59 $(95 \%$ Cl: 0.37-0.92)) within the chemotherapy cohort. Obese patients had a significantly longer progression-free survival (PFS) (HR=0.61 (95\% Cl: 0.45-0.82)) in the pembrolizumab cohort. Conversely, they had a significantly shorter PFS in the chemotherapy cohort ( $\mathrm{HR}=1.27(95 \%$ $\mathrm{Cl}: 1.01-1.60))$. Obese patients had a significantly longer overall survival (OS) within the pembrolizumab cohort ( $\mathrm{HR}=0.70$ (95\% Cl: 0.49-0.99)), while no significant differences according to baseline BMI were found in the chemotherapy cohort. BMI variation significantly affected
ORR, PFS and OS in both the pembrolizumab and the chemotherapy cohorts.

Conclusions Baseline obesity is associated to significantly improved ORR, PFS and OS in metastatic NSCLC patients with a PD-L1 expression of $\geq 50 \%$, receiving first line pembrolizumab, but not among patients treated with chemotherapy. BMI variation is also significantly related to clinical outcomes.

\section{INTRODUCTION}

Obesity-associated inflammation has been shown to dysregulate the immune response, potentially having profound effects on the toxicity and efficacy of immunotherapy across different type of malignancies. ${ }^{1-5}$ In non-small cell lung cancer (NSCLC), recent evidences have already confirmed the positive association between obesity and improved outcomes in patients receiving immune checkpoint inhibitors as further line of therapy, regardless of programmed death ligand-1 (PD-L1) tumor expression. ${ }^{6}$ Moreover, a pooled post-hoc analysis of data from four prospective trials (two of which randomized, with docetaxel as control arm) of pre-treated NSCLC patients receiving atezolizumab has provided robust evidences supporting this hypothesis. ${ }^{8}$ Importantly, Kichenadasse 
and colleagues confirmed that obesity was associated to survival benefit (compared with normal-weight patients) in patients treated with atezolizumab, but not in those who received chemotherapy, suggesting a predictive as well as a prognostic role of a high body mass index (BMI) ${ }^{8}$ Of note, the survival benefit was more pronounced in the PD-L1-positive subgroup, while a loss of statistical significance was reported within the PD-L1-negative patients. ${ }^{8}$

Accordingly, some evidences suggest that adipose tissue might play a pivotal role in regulating the immune homeostasis. ${ }^{9}{ }^{10}$ In a recent meta-analysis including 203 articles, more than 6,000,000 patients across 15 different malignancies, the association between obesity and improved clinical outcomes was confirmed in those tumor types in which programmed death-1 (PD-1)/PD-L1 checkpoint inhibitors have proven to be more effective (such as lung cancer and renal cell carcinoma), despite studies involving patients who received immune checkpoint inhibitors were poorly represented in the meta-analysis itself. ${ }^{11}$ Therefore, assuming that the adipose tissue has somehow a role in the antitumor immune response, evaluating whether the variation of the BMI in patients receiving immune checkpoint inhibitors affects the clinical outcomes represents an interesting area of research.

Recently, we published a large real-world multicenter study of metastatic NSCLC patients with a PD-L1 expression $\geq 50 \%$, receiving first line single agent pembrolizumab at 34 European institution, aimed at investigating the clinic-pathologic correlates of efficacy. ${ }^{12}$ Here, we present the clinical outcomes analysis according to baseline BMI and BMI variation (defined as $\triangle \mathrm{BMI}$ ) of the same study population. In order to confirm the results, we also evaluated a control cohort of metastatic NSCLC patients treated with first line platinum-based chemotherapy in clinical practice.

\section{MATERIALS AND METHODS Study design}

We assessed baseline BMI and $\triangle \mathrm{BMI}$ within the study population of a real-world multicenter retrospective study evaluating metastatic NSCLC patients with PD-L1 expression $\geq 50 \%$, consecutively treated with first line pembrolizumab monotherapy, from January 2017 to October 2019, at 34 institutions (online supplemental table 1). ${ }^{13}$ In order to weighing our results, we also evaluated the baseline BMI and $\triangle \mathrm{BMI}$ in a cohort of metastatic epidermal growth factor receptor wild-type NSCLC patients treated with platinum-based doublet chemotherapy in clinical practice from January 2013 to January 2020, at 10 among the above-mentioned institutions. Patients were treated in clinical practice with either the $2 \mathrm{mg} / \mathrm{kg}$ q3 weeks schedules (before the procedure $\mathrm{N}^{\circ}$ EMEA/H/C/3820/II/48) and the $200 \mathrm{mg}$ q 3 weeks flat dose.

The aim of this analysis was to evaluate clinical outcomes according to baseline BMI and $\triangle \mathrm{BMI}$ in both the pembrolizumab and chemotherapy cohorts. The measured clinical outcomes were objective response rate (ORR), median progression-free survival (PFS) and median overall survival (OS). Patients were assessed with radiological imaging in clinical practice, with a frequency ranging from 12 to 16 weeks, according to the monitoring requirements for high-cost drugs of the respective national drug regulatory agencies (eg, the on-line monitoring dashboard of the 'Agenzia Italiana del Farmaco' requires a disease assessment at least every 16 weeks; available at: https://servizionline.aifa.gov.it/). Radiologists evaluation was based on Response Evaluation Criteria In Solid Tumors (RECIST criteria (V.1.1), ${ }^{14}$ and a subsequent confirming imaging was recommended. However, treatment beyond disease progression was allowed and also patients evaluated according to the clinicians assessment in clinical practice were considered eligible. The reliability of disease response evaluation in clinical practice was assessed through Kaplan-Meier survival curves for PFS and OS according to the best response (categorized as partial/complete response, stable disease and progressive disease) (online supplemental figure 1).

ORR was defined as the portion of patients experiencing an objective response (complete or partial response) as best response to immunotherapy. PFS was defined as the time from treatment initiation to disease progression or death, whichever occurred first. OS was defined as the time from treatment initiation to death. For PFS as well as for OS, patients without events were considered as censored at the time of the last follow-up. Data cut-off period was February 2020 for the pembrolizumab cohort and April 2020 for the chemotherapy cohort.

Considering the possible unbalanced distribution, the influence of large within group variation and the possible interactions, fixed multivariable regression models were used to estimate clinical outcomes (ORR, PFS and OS) according to baseline BMI and $\triangle \mathrm{BMI}$ by using pre-planned adjusting key covariates in both the pembrolizumab and chemotherapy cohorts. ${ }^{15-17}$ The key covariates were: age $\left(<70\right.$ vs $\geq 70$ years old),${ }^{18}$ gender (male vs female), Eastern Cooperative Oncology Group-Performance Status (ECOG-PS) (0-1 vs $\geq 2)$, central nervous system (CNS) metastases (yes vs no), bone metastases (yes vs no) and liver metastases (yes vs no).

\section{BMI evaluation}

Weight and height were obtained from patient medical records; BMI was calculated using the formula of weight/ height $^{2}$ (kilograms per square meter) and categorized according to the $\mathrm{WHO}$ categories: underweight, $\mathrm{BMI}<18.5$; normal-weight, $18.5 \leq \mathrm{BMI} \leq 24.9$; overweight, $25 \leq \mathrm{BMI} \leq 29.9$; obese, $\mathrm{BMI} \geq 30$. In all the regression analyses, normal-weight patients were considered as the comparator group. $\triangle \mathrm{BMI}$ was defined as the BMI percentage variation from treatment initiation to disease progression (or to the last contact for censored patients). In order to guarantee a minimum time lapse for $\Delta \mathrm{BMI}$ assessment and to overcome selection biases related to some fast disease progressions, which might have flawed our determinations, the efficacy analysis according to the 
$\triangle \mathrm{BMI}$ was performed after a 4 weeks landmark selection, including only patients with a minimum follow-up for PFS of 4 weeks. To identify an optimal grouping according to the $\triangle \mathrm{BMI}$ and determine appropriate cut-offs with respect to ORR, PFS and OS in the pembrolizumab, a recursive partitioning algorithm was performed, using the Rpart function in R. The computed cut-offs were than applied to the chemotherapy cohort, following the rules of external validation. ${ }^{19}$ However, in order to properly weighing the role of the $\Delta \mathrm{BMI}$, a recursive partitioning was performed

Table 1 Patients' characteristics

\begin{tabular}{|c|c|c|c|c|c|c|}
\hline & $\begin{array}{l}\text { Pembrolizumab } \\
\text { cohort } \\
962 n(\%)\end{array}$ & $\begin{array}{l}\text { Chemotherapy } \\
\text { cohort } \\
426 n(\%)\end{array}$ & $\chi^{2}$ test & $\begin{array}{l}\text { Pembrolizumab } \\
\text { cohort (4 weeks } \\
\text { landmark) } \\
799 \mathrm{n}(\%)\end{array}$ & $\begin{array}{l}\text { Chemotherapy } \\
\text { cohort (4 weeks } \\
\text { landmark) } \\
414 \mathrm{n}(\%)\end{array}$ & $\chi^{2}$ test \\
\hline \multicolumn{7}{|l|}{ Age (years) } \\
\hline Median & 70.1 & 66.0 & & 70.2 & 66.0 & \\
\hline Range & 28-92 & $24-85$ & $p<0.0001$ & 28-92 & $24-84$ & $p=0.0002$ \\
\hline Elderly $(\geq 70)$ & $491(51.0)$ & 167 (39.2) & & 410 (51.3) & 165 (39.9) & \\
\hline \multicolumn{7}{|l|}{ Smoking status } \\
\hline Former/current & $864(89.8)$ & $378(88.7)$ & $p=0.5452$ & 719 (90.0) & 369 (89.1) & $p=0.6417$ \\
\hline Never & $98(10.2)$ & 48 (11.3) & & $80(10.0)$ & 45 (10.9) & \\
\hline \multicolumn{7}{|l|}{ Sex } \\
\hline Male & $635(66.0)$ & $295(69.2)$ & $p=0.2365$ & $535(67.0)$ & 288 (69.6) & $p=0.3569$ \\
\hline Female & $327(34.0)$ & $131(30.8)$ & & $264(33.0)$ & $126(30.4)$ & \\
\hline \multicolumn{7}{|l|}{ ECOG PS } \\
\hline $0-1$ & 793 (82.4) & 396 (93.0) & $p<0.0001$ & $685(85.7)$ & 385 (93.0) & $p=0.0002$ \\
\hline$\geq 2$ & 169 (17.6) & $30(7.0)$ & & 114 (14.3) & $29(7.0)$ & \\
\hline \multicolumn{7}{|l|}{ Histology } \\
\hline Squamous & $232(24.1)$ & 95 (22.3) & $p=0.4623$ & 188 (23.5) & 95 (22.9) & $p=0.8201$ \\
\hline Non-squamous & 730 (75.9) & $331(77.7)$ & & $611(76.5)$ & 319 (77.1) & \\
\hline \multicolumn{7}{|l|}{ CNS metastases } \\
\hline Yes & $171(17.8)$ & $67(15.7)$ & $p=0.3507$ & 138 (17.3) & 66 (15.9) & $p=0.5574$ \\
\hline No & 791 (82.2) & $359(84.3)$ & & $661(82.7)$ & $348(84.1)$ & \\
\hline \multicolumn{7}{|l|}{ Bone metastases } \\
\hline Yes & $310(32.2)$ & $115(27.0)$ & $\mathrm{p}=0.0513$ & $240(30.0)$ & $112(27.1)$ & $p=0.2777$ \\
\hline No & $652(67.8)$ & $311(73.0)$ & & $559(70.0)$ & 302 (72.9) & \\
\hline \multicolumn{7}{|l|}{ Liver metastases } \\
\hline Yes & $150(15.7)$ & $57(13.4)$ & $p=0.2730$ & 119 (14.9) & $56(13.5)$ & $p=0.5207$ \\
\hline No & 808 (84.3) & 369 (86.6) & & $680(85.1)$ & $358(86.5)$ & \\
\hline \multicolumn{7}{|l|}{$\mathrm{BMI}\left(\mathrm{kg} / \mathrm{m}^{2}\right)$} \\
\hline Median (range) & $24.2(14.0-44.9)$ & $24.9(15.7-41.8)$ & & $24.2(14.0-44.9)$ & $24.9(15.7-41.8)$ & \\
\hline $\begin{array}{l}\text { Underweight } \\
(\leq 18.5)\end{array}$ & $40(4.2)$ & $16(3.8)$ & $p=0.0505$ & $40(4.2)$ & $10(2.4)$ & $p=0.0210$ \\
\hline $\begin{array}{l}\text { Normal weight } \\
(18.5-25)\end{array}$ & $526(54.9)$ & 202 (47.4) & $\mathrm{p}=0.0287^{*}$ & $526(54.9)$ & $200(48.3)$ & $p=0.0089$ \\
\hline $\begin{array}{l}\text { Overweight } \\
(25-30)\end{array}$ & 275 (28.7) & $150(35.2)$ & & 275 (28.7) & 147 (35.5) & \\
\hline Obese $(\geq 30)$ & 117 (12.2) & $58(13.6)$ & & 117 (12.2) & $57(13.8)$ & \\
\hline$\Delta \mathrm{BMI}$ (sample) & - & - & & (799) (83.1) & (414) (97.2) & \\
\hline Median & - & - & & $0 \%$ & $0 \%$ & \\
\hline Range & & & & $(-34 \%$ to $+34 \%)$ & $(-22 \%$ to $+27 \%)$ & \\
\hline
\end{tabular}

${ }^{*} \chi^{2}$ test for trend.

BMI, body mass index; CNS, central nervous system; ECOG-PS, Easter Cooperative Oncology Group-Performance Status. 
Table 2 Optimal grouping according to $\triangle \mathrm{BMI}$ computed through the recursive partitioning algorithm with respects to the objective response rate (ORR), progression-free survival (PFS) and overall survival (OS)

\begin{tabular}{|c|c|c|c|c|c|c|}
\hline & \multicolumn{3}{|c|}{ Pembrolizumab cohort } & \multicolumn{3}{|c|}{ Chemotherapy cohort } \\
\hline & ORR & PFS & OS & ORR & PFS & os \\
\hline$\triangle \mathrm{BMI}(\%)$ computed cut-off & +1.4 & +1.4 & +1.4 & +7.1 & -6.8 & -2.2 \\
\hline$\geq$ cut off & $173(24.0 \%)$ & $183(22.9 \%)$ & $183(22.9 \%)$ & $36(8.8 \%)$ & 345 (83.3\%) & $266(64.3 \%)$ \\
\hline$<$ cut-off & 548 (76.0\%) & 616 (77.1\%) & 616 (77.1\%) & 375 (91.2\%) & 69 (16.7\%) & 148 (35.7\%) \\
\hline
\end{tabular}

BMI, body mass index.

to compute the cut-offs with respect to ORR, PFS and OS also within the chemotherapy cohort.

\section{Statistical analysis}

Baseline patient characteristics were reported with descriptive statistics and compared between the pembrolizumab cohort and chemotherapy cohort with the $\chi^{2}$ test. $\chi^{2}$ test was also used to compare ORRs according to BMI and $\triangle \mathrm{BMI}$ in both the cohorts. Logistic regression was used for the multivariate analysis of ORR. Median PFS and median OS were evaluated using the Kaplan-Meier method. Median period of follow-up was calculated according to the reverse Kaplan-Meier method. Cox proportional hazards regression was used for the fixed multivariate analysis of PFS and OS. The alpha level for all analyses was set to $\mathrm{p}<0.05$. Adjusted hazard ratios (aHRs) and adjusted odds ratios (aORs) with 95\% CIs were calculated. Forest plot graphs were used to compare HRs and ORs between the pembrolizumab and the chemotherapy cohorts. All statistical analyses were performed using MedCalc Statistical Software V.18.11.3 (MedCalc Software bvba, Ostend, Belgium; http://www.medcalc.org; 2019). Recursive partitioning was performed using the $R$ package rpart (R V.3.6.2).

\section{RESULTS \\ Patients characteristics}

Table 1 summarizes patients characteristics of both the cohorts. Nine hundred and sixty-two patients and 426 patients were included in the pembrolizumab and chemotherapy cohorts, respectively. Median age was 70.1 and 66.0 years; the rate of elderly patients was significantly higher in the pembrolizumab cohort, compared with the chemotherapy cohort ( $51.0 \%$ vs $39.2 \%, \mathrm{p}<0.0001)$, as well as the rate of patients with an ECOG-PS of $\geq 2(17.6 \%$ vs $7.0 \%, \mathrm{p}<0.0001)$. The median baseline BMI was 24.2 for the pembrolizumab cohort and 24.9 for the chemotherapy cohort; normal-weight patients were the majority of both the cohort (54.9\% and $47.4 \%$, respectively), but the $\chi^{2}$ test for trend revealed that there was a statistically significant trend of higher BMI categories to be more frequent through the chemotherapy cohort $(\mathrm{p}=0.0287)$. After the 4 weeks landmark selection, $799(83.1 \%)$ and $414(97.2 \%)$ of the patients from the pembrolizumab and chemotherapy cohorts were included in the $\triangle \mathrm{BMI}$ analysis, respectively (online supplemental figure 2). The median $\triangle \mathrm{BMI}$ was $0 \%$ for both the cohorts. Notably, the median follow-up periods were 14.6 months (95\% CI: 13.5-15.6) and 37.2 months (95\% CI: 31.7-44.1) for the

Table 3 Summary of the objective response rate (ORR) in the pembrolizumab cohort and chemotherapy cohort according to the baseline $B M I$ and $\triangle \mathrm{BMI}$

\begin{tabular}{|c|c|c|c|c|c|c|}
\hline & \multicolumn{6}{|c|}{ Objective response rate } \\
\hline & \multicolumn{2}{|c|}{ Pembrolizumab cohort } & \multirow[b]{2}{*}{$\chi^{2}$ test } & \multicolumn{2}{|c|}{ Chemotherapy cohort } & \multirow[b]{2}{*}{$\chi^{2}$ test } \\
\hline & $\begin{array}{l}\text { Response- } \\
\text { ratio }\end{array}$ & ORR (\%) $(95 \% \mathrm{Cl})$ & & $\begin{array}{l}\text { Response- } \\
\text { ratio }\end{array}$ & ORR (\%) $(95 \% \mathrm{Cl})$ & \\
\hline \multicolumn{7}{|l|}{$\operatorname{BMI}\left(\mathrm{kg} / \mathrm{m}^{2}\right)$} \\
\hline Underweight $(\leq 18.5)$ & $10 / 35$ & $28.6(13.7-52.5)$ & \multirow{4}{*}{$\begin{array}{l}p=0.0077 \\
p=0.0910^{*}\end{array}$} & $5 / 15$ & $33.3(10.8-77.8)$ & \multirow{4}{*}{$\begin{array}{l}p=0.0758 \\
p=0.3079^{*}\end{array}$} \\
\hline Normal weight (18.5-25) & $201 / 467$ & $43.0(37.3-49.4)$ & & $96 / 199$ & $48.2(39.1-58.9)$ & \\
\hline Overweight (25-30) & $97 / 232$ & $41.8(33.9-51.0)$ & & $54 / 149$ & $36.2(27.2-47.3)$ & \\
\hline Obese $(\geq 30)$ & $61 / 106$ & $57.5(44.0-73.9)$ & & $29 / 57$ & $50.9(34.1-73.1)$ & \\
\hline \multicolumn{7}{|l|}{$\triangle \mathrm{BMI}$} \\
\hline$\geq 1.4 \%$ & $116 / 173$ & $67.1(55.4-80.4)$ & \multirow[t]{2}{*}{$\mathrm{p}<0.0001$} & $72 / 119$ & $60.5(47.3-76.2)$ & \multirow[t]{2}{*}{$\mathrm{p}<0.0001$} \\
\hline$<1.4 \%$ & $227 / 548$ & $41.4(36.2-47.2)$ & & 111/292 & $38.0(31.2-45.8)$ & \\
\hline
\end{tabular}

${ }^{*} \chi^{2}$ test for trend.

BMI, Body mass index. 


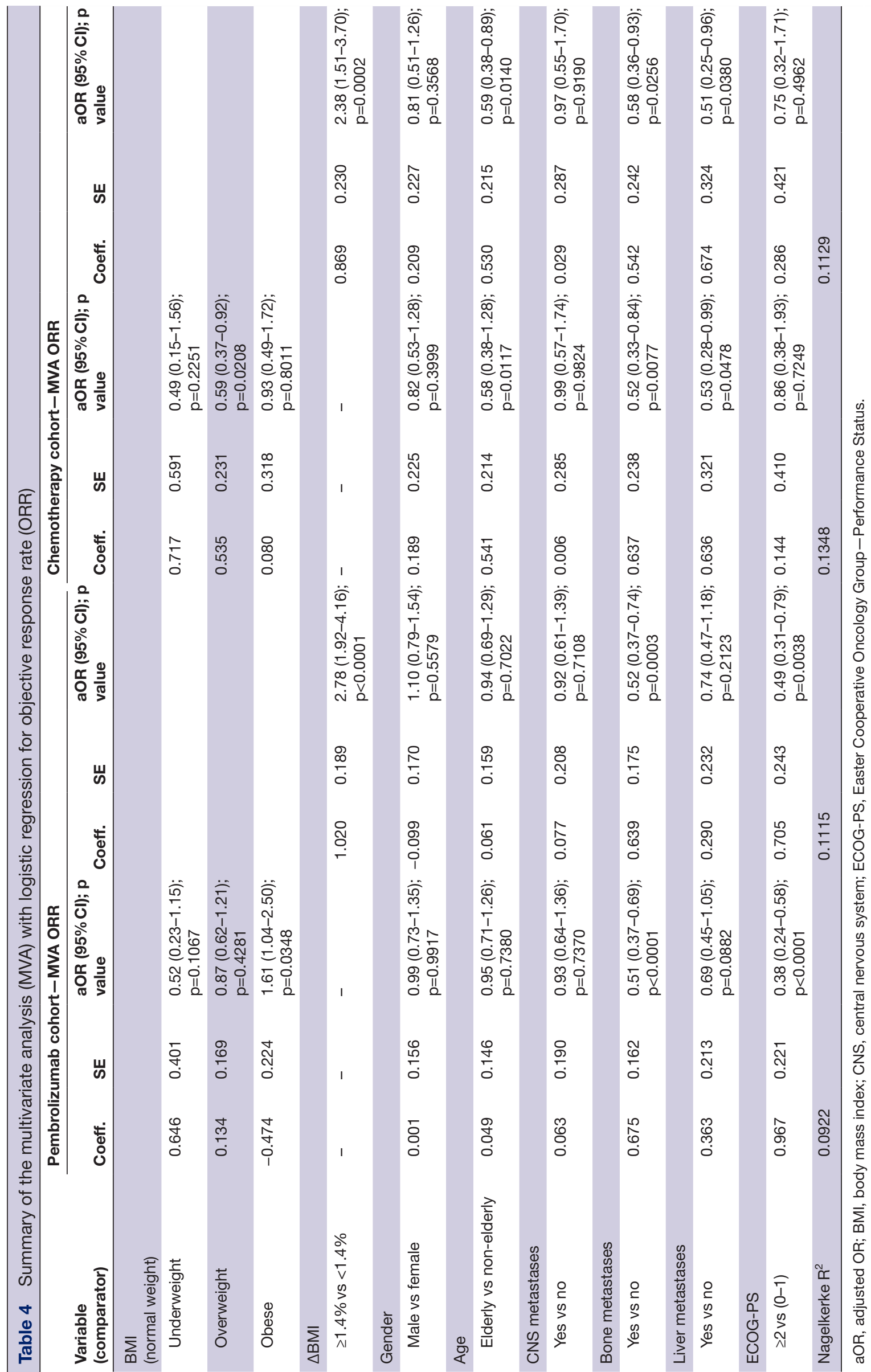


ORR, aOR for disease response $(95 \% \mathrm{Cl})$

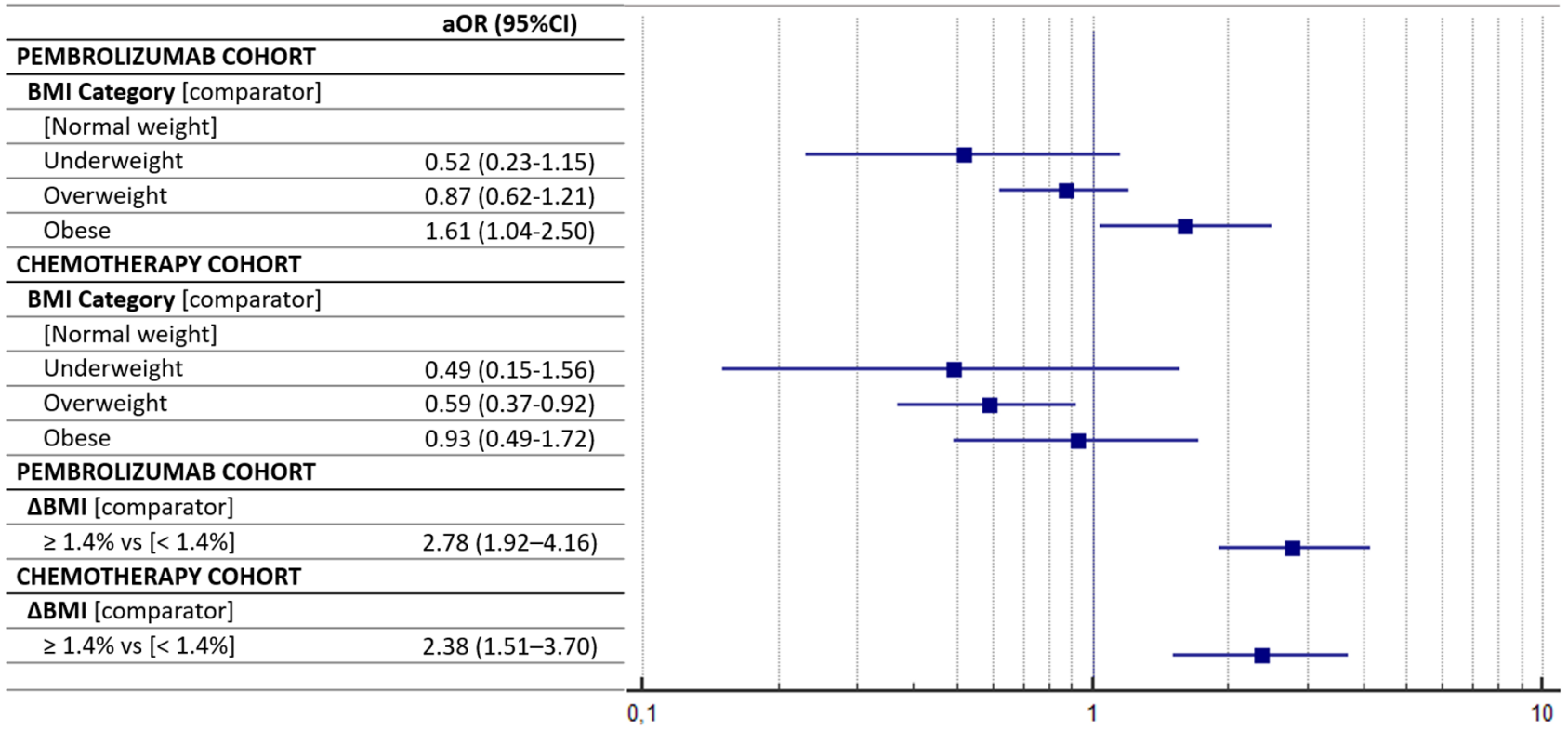

Figure 1 Forest plot graph for objective response rate (ORR). aOR, adjusted OR; BMI, body mass index.

pembrolizumab and chemotherapy cohorts, respectively. One hundred ninety-three patients $(20 \%)$ within the pembrolizumab cohort had received a further systemic treatment, while 249 patients $(58.5 \%)$ within the chemotherapy cohort had received a further treatment with either PD-1 or PD-L1 checkpoint inhibitors at the data cut-off. Table 2 summarizes the recursive partitioning results. Interestingly, the computed cut-offs for ORR, PFS and OS were the same positive value (BMI gain of $1.4 \%$ ) within the pembrolizumab cohort, which was set as fixed cut-off for the clinical outcomes analysis of both the cohorts. On the other hand, in the chemotherapy cohort the computed cut-off for ORR was a positive value (BMI gain of $7.1 \%$ ), while was a negative value for PFS and OS (BMI loss of $6.8 \%$ and $2.2 \%$, respectively).

\section{Clinical outcomes analysis}

The ORR for the pembrolizumab cohort overall was 44.1\% (95\% CI: 39.7-48.8) (372/844 response ratio), while was $43.8 \%$ for the chemotherapy cohort overall (95\% CI: 37.7-50.6) (184/420 response ratio). The median PFS and OS for the pembrolizumab cohort were 8.0 months (95\% CI: 6.9-9.6; 559 progression events) and 18.6 months (95\% CI: 16.1-27.5; 567 censored patients), respectively, while the median PFS and OS for the chemotherapy cohort were 6.1 months (95\% CI: 5.7-6.5; 387 progression events) and 16.5 months (95\% CI: 13.6-18.7; 136 censored), respectively (online supplemental figure 3 ). Table 3 summarizes the ORRs of both the pembrolizumab and chemotherapy cohorts, according to the baseline BMI and $\triangle \mathrm{BMI}$ of $1.4 \%$. Patients with a high baseline BMI had a significantly higher ORR within the pembrolizumab cohort $(\mathrm{p}=0.0077)$, but not within the chemotherapy cohort $(\mathrm{p}=0.0758)$. The $\Delta \mathrm{BMI}$ evaluated according to the computed cut-off significantly affected the ORR and patients with a BMI gain of $\geq 1.4 \%$ had a higher BMI in both the pembrolizumab $(\mathrm{p}<0.0001)$ and the chemotherapy $(\mathrm{p}<0.0001)$ cohorts. Table 4 summarizes the multivariate regression analyses for ORR with the pre-planned fixed adjusting factors. Obese patients were confirmed to have a significantly higher ORR compared with normal-weight patients $(\mathrm{aOR}=1.61 \quad(95 \%$ CI: $1.04-2.50), \mathrm{p}=0.0348$ ) within the pembrolizumab cohort, while overweight patients had a significantly lower ORR compared with normal-weight patients $(\mathrm{aOR}=0.59$ (95\% CI: $0.37-0.92), p=0.0208$ ) within the chemotherapy cohort. Also, at the multivariate analysis. the $\triangle \mathrm{BMI}$ of $1.4 \%$ significantly affected the ORR within the pembrolizumab cohort $(\mathrm{aOR}=2.78$ (95\% CI: 1.92-4.16), $\mathrm{p}<0.0001)$ and the chemotherapy cohort $(\mathrm{aOR}=2.38$ (95\% CI: $1.51-$ $3.70), p=0.0002$ ). Figure 1 reports the forest plot graph for the ORR of the pembrolizumab and chemotherapy cohorts according to baseline BMI and $\triangle \mathrm{BMI}$.

Table 5 summarizes the multivariate regression for PFS. Obese patients were confirmed to have a significantly longer PFS compared with normal-weight patients $\quad(\mathrm{aHR}=0.61 \quad(95 \%$ CI: 0.45-0.82), $\mathrm{p}=0.0012)$ in the pembrolizumab cohort. Conversely, they had a significantly shorter PFS compared with normal-weight patients in the chemotherapy cohort $(\mathrm{aHR}=1.37(95 \%$ CI: $1.01-1.87), \mathrm{p}=0.0477)$. $\Delta \mathrm{BMI}$ of $1.4 \%$ significantly affected the PFS of both the pembrolizumab cohort $(\mathrm{aHR}=0.39 \quad(95 \%$ CI: $0.29-0.51), \mathrm{p}<0.0001)$ and the chemotherapy cohort (aHR $=0.78$ (95\% CI: $0.62-0.99$ ), $\mathrm{p}=0.0341$ ). Figure 2 reports the forest plot for the PFS of the two cohort according to baseline BMI and $\triangle \mathrm{BMI}$.

Table 6 summarizes the multivariate regression for OS. Obese patients had a significantly longer OS compared with normal-weight patients within the pembrolizumab 
Table 5 Summary of the multivariate analysis (MVA) with Cox proportional-hazards of progression-free survival (PFS)

\begin{tabular}{|c|c|c|c|c|}
\hline \multirow{2}{*}{$\begin{array}{l}\text { Variable } \\
\text { (comparator) }\end{array}$} & \multicolumn{2}{|c|}{ Pembrolizumab cohort-MVA PFS } & \multicolumn{2}{|c|}{ Chemotherapy cohort-MVA PFS } \\
\hline & aHR $(95 \% \mathrm{Cl}) ; \mathrm{p}$ value & aHR $(95 \% \mathrm{Cl}) ; \mathrm{p}$ value & aHR $(95 \% \mathrm{CI}) ; \mathrm{p}$ value & aHR $(95 \% \mathrm{Cl}) ; \mathrm{p}$ value \\
\hline \multicolumn{5}{|l|}{$\begin{array}{l}\text { BMI (normal } \\
\text { weight) }\end{array}$} \\
\hline Underweight & $\begin{array}{l}1.46(0.98-2.20) \\
p=0.0625\end{array}$ & & $\begin{array}{l}0.97(0.56-1.69) \\
p=0.9332\end{array}$ & \\
\hline Overweight & $\begin{array}{l}1.04(0.85-1.26) \\
p=0.6736\end{array}$ & & $\begin{array}{l}0.95(0.76-1.20) \\
p=0.7239\end{array}$ & \\
\hline Obese & $\begin{array}{l}0.61(0.45-0.82) \\
p=0.0012\end{array}$ & & $\begin{array}{l}1.37(1.01-1.87) \\
\mathrm{p}=0.0477\end{array}$ & \\
\hline \multicolumn{5}{|l|}{$\triangle \mathrm{BMI}$} \\
\hline$\geq 1.4 \%$ vs $<1.4 \%$ & - & $\begin{array}{l}0.39(0.29-0.51) \\
p<0.0001\end{array}$ & - & $\begin{array}{l}0.78(0.62-0.99) \\
p=0.0341\end{array}$ \\
\hline \multicolumn{5}{|l|}{ Age } \\
\hline $\begin{array}{l}\text { Elderly vs non- } \\
\text { elderly }\end{array}$ & $\begin{array}{l}1.08(0.91-1.28) \\
p=0.3606\end{array}$ & $\begin{array}{l}1.13(0.92-1.37) \\
p=0.2190\end{array}$ & $\begin{array}{l}1.20(0.97-1.48) \\
p=0.0810\end{array}$ & $\begin{array}{l}1.14(0.92-1.41) \\
p=0.2221\end{array}$ \\
\hline \multicolumn{5}{|l|}{ CNS metastases } \\
\hline Yes vs no & $\begin{array}{l}1.25(1.01-1.55) \\
p=0.0366\end{array}$ & $\begin{array}{l}1.18(0.92-1.51) \\
p=0.1881\end{array}$ & $\begin{array}{l}1.15(0.86-1.54) ; \\
p=0.3222\end{array}$ & $\begin{array}{l}1.12(0.85-1.49) \\
p=0.4139\end{array}$ \\
\hline \multicolumn{5}{|l|}{ Bone metastases } \\
\hline Yes vs no & $\begin{array}{l}1.62(1.36-1.94) \\
p<0.0001\end{array}$ & $\begin{array}{l}1.74(1.42-2.13) \\
p<0.0001\end{array}$ & $\begin{array}{l}1.25(1.01-1.57) \\
p=0.0460\end{array}$ & $\begin{array}{l}1.22(0.98-1.53) \\
p=0.0729\end{array}$ \\
\hline \multicolumn{5}{|l|}{ ECOG-PS } \\
\hline
\end{tabular}

aHR, adjusted HR; BMI, body mass index; CNS, central nervous system; ECOG-PS, Easter Cooperative Oncology Group-Performance Status.

cohort $(\mathrm{aHR}=0.70$ (95\% CI: 0.49-0.99)), while no significant differences according to baseline BMI were found in the chemotherapy cohort. $\triangle \mathrm{BMI}$ of $1.4 \%$ significantly affected the OS within both the pembrolizumab cohort $(\mathrm{aHR}=0.33$ (95\% CI: 0.22-0.48), $\mathrm{p}<0.0001)$ and the chemotherapy cohort $(\mathrm{aHR}=0.73$ (95\% CI: $0.56-0.96), \mathrm{p}=0.0241)$. Figure 3 reports the forest plot graph for the OS of the two cohorts according to baseline BMI and $\triangle \mathrm{BMI}$.

Online supplemental figure 4 reports the Kaplan-Meier survival curves of PFS and OS according to baseline BMI; online supplemental figure 5 reports the Kaplan-Meier survival curves of PFS and OS according to the $\triangle \mathrm{BMI}$ for both the cohorts.

\section{DISCUSSION}

In this study we confirmed that also in a population of NSCLC patients with a PD-L1 expression $\geq 50 \%$ receiving first line pembrolizumab, obesity is associated to improved clinical outcomes. Obese patients had a significantly higher ORR, and prolonged PFS and OS compared with normal-weight patients. The fact that this trend was confirmed at each outcome analysis (ORR, PFS and OS) and with multivariate regression models further supports our results. More importantly, we did not observe such correlation among patients treated with chemotherapy, suggesting that the obesity-related clinical benefit is unique of patients treated with immunotherapy. Of note, obese patients who received chemotherapy had a nonsignificant trend towards a lower ORR and shorter OS, and a significantly shorter PFS, compared with normal-weight patients. These evidences are aligned to what already reported for NSCLC patients in clinical practice, ${ }^{6720}$ and to the improved survival observed in obese PD-L1-positive NSCLC patients by Kichenadasse et $a l^{8}$ Consistently with our results, also in the control cohort of the study by Kichenadasse and colleagues, no survival benefit was 
PFS; aHR for disease progression $(95 \% \mathrm{Cl})$

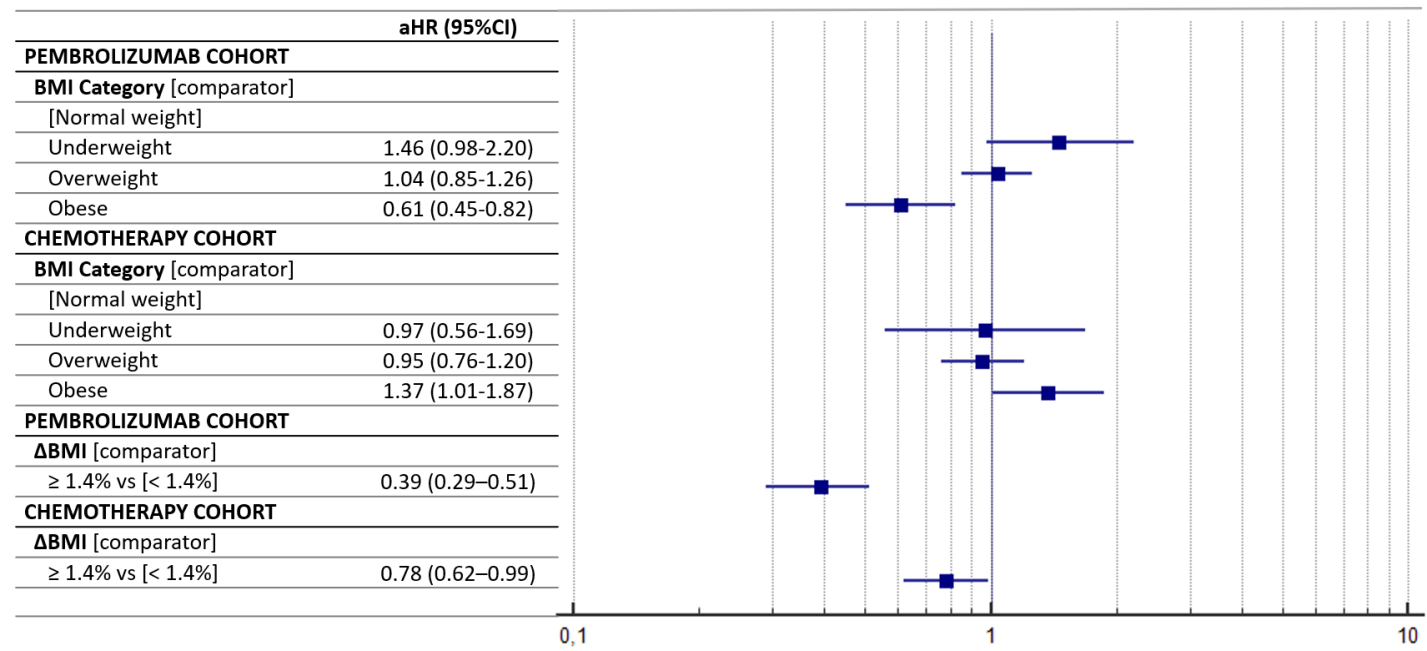

Figure 2 Forest plot graph for progression-free survival (PFS). aHR, adjusted HR; BMI, body mass index.

reported for obese patients who received chemotherapy. ${ }^{8}$ Remarkably, obese patients in the chemotherapy cohort did not have an improved OS despite $58.5 \%$ of them received immunotherapy on chemotherapy failure.

As previously mentioned, a growing body of evidence is indicating that the adipose tissue might play a critical role in shaping the antitumor immune responses. Wang and colleagues reported that obese mice showed a significant increase of dysfunctional exhausted T-cell. ${ }^{9}$ Such exhaustion could be partially mediated by the immune checkpoints (as the PD-1/PD-L1 pathway) and driven by leptin, and then might be more likely elicited by immune checkpoints inhibitors. ${ }^{910}$

Nonetheless, a high baseline BMI has been historically somehow associated to improved lung cancer survival across different disease stages, even in the 'preimmune checkpoint inhibitors era', reflecting the potential prognostic nature of baseline BMI in lung cancer patients. ${ }^{21-24}$ However, we need to highlight that even if cancer cachexia mechanisms are not completely known yet, several evidences showed that the systemic inflammation plays a central role. ${ }^{25}$ Lung cancer is an aggressive disease, and metastatic NSCLC patients usually to present with poor clinical condition and weight loss at the time of first line treatment commencement, which underlies a systemic inflammatory over-activation. ${ }^{26}$ From this point of view, a higher baseline BMI might be considered a sign of functional reserve and a protective feature for NSCLC advanced patients.

To that end, in a study evaluating 2585 stage IV NSCLC patients from three trials with first line chemotherapy, obesity was associated with improved OS. ${ }^{27}$ Intriguingly, when time on-study exceeded 16 months, obese patients experienced a significant increase in their hazard rate for death, compared with normal/overweight patients, suggesting a possible negative role of the sarcopenic obesity in the long-term during chemotherapy. ${ }^{27}$
Also, the efficacy analysis according to $\triangle \mathrm{BMI}$ revealed interesting results. A BMI variation of $1.4 \%$ during treatment was significantly (and concordantly) related to each measured outcome (ORR, PFS and OS) for both the pembrolizumab and chemotherapy cohorts. Looking to the forest plot graphs, we can notice that the aOR and the aHRs were concordantly more pronounced for the pembrolizumab cohort. Similarly, the Kaplan-Meier survival curves according to the $\triangle \mathrm{BMI}$ (online supplemental figure 4) show a much more marked survival benefit for patients who experienced a BMI gain of $\geq 1.4 \%$ within the pembrolizumab cohort rather than in the chemotherapy cohort. These findings could make us assuming that the prognostic role $\Delta \mathrm{BMI}$ is stronger during the immunotherapy rather than chemotherapy. However, we must not fail in taking into account that the optimal grouping computed with the recursive partitioning algorithm within the patients receiving pembrolizumab was applied to both the cohorts. Interestingly with respect to the optimal grouping, while for the pembrolizumab cohort a harmonic positive cut-off for $\Delta \mathrm{BMI}$ was found for ORR, PFS and OS $(+1.4 \%)$, three different cutoffs were found among the chemotherapy cohort using a recursive partitioning: a positive value for ORR $(+7.1 \%)$, and two different negative values for PFS $(-6.8 \%)$ and OS $(-2.2 \%)$. Although using different cut-offs for the same variable may seem redundant, the results raise some questions. A harmonic and relatively small weight gain during pembrolizumab could be considered a concordant independent predictor for ORR, PFS and OS. Conversely, a more pronounced weight gain during chemotherapy is associated with ORR, while a weight loss is significantly associated with inferior PFS and OS. From this perspective, the BMI acts differently in the two cohorts; considering the adipose tissue an immune organ, patients who experienced a slight weight gain during immunotherapy had a significantly improved ORR, PFS and OS. Considering 
Table 6 Summary of the multivariate analysis (MVA) with Cox proportional-hazards of overall survival (OS)

\begin{tabular}{|c|c|c|c|c|}
\hline \multirow{2}{*}{$\begin{array}{l}\text { Variable } \\
\text { (comparator) }\end{array}$} & \multicolumn{2}{|c|}{ Pembrolizumab cohort-MVA OS } & \multicolumn{2}{|c|}{ Chemotherapy cohort-MVA OS } \\
\hline & aHR $(95 \% \mathrm{Cl}) ; \mathrm{p}$ value & aHR $(95 \% \mathrm{Cl}) ;$ p value & aHR $(95 \% \mathrm{Cl}) ; \mathrm{p}$ value & aHR $(95 \% \mathrm{Cl}) ; \mathrm{p}$ value \\
\hline \multicolumn{5}{|l|}{$\begin{array}{l}\text { BMI (normal } \\
\text { weight) }\end{array}$} \\
\hline Underweight & $\begin{array}{l}1.22(0.74-2.01) \\
p=0.4309\end{array}$ & & $\begin{array}{l}0.69(0.36-1.30) \\
p=0.2513\end{array}$ & \\
\hline Overweight & $\begin{array}{l}0.97(0.77-1.22) \\
p=0.8100\end{array}$ & & $\begin{array}{l}0.82(0.63-1.08) \\
p=0.1704\end{array}$ & \\
\hline Obese & $\begin{array}{l}0.70(0.49-0.99) \\
\mathrm{p}=0.0474\end{array}$ & - & $\begin{array}{l}1.29(0.89-1.86) \\
p=0.1763\end{array}$ & - \\
\hline \multicolumn{5}{|l|}{$\triangle \mathrm{BMI}$} \\
\hline$\geq 1.4 \%$ vs $<1.4 \%$ & - & $\begin{array}{l}0.33(0.22-0.48) \\
p<0.0001\end{array}$ & - & $\begin{array}{l}0.73(0.56-0.96) \\
p=0.0241\end{array}$ \\
\hline \multicolumn{5}{|l|}{ Age } \\
\hline $\begin{array}{l}\text { Elderly vs non- } \\
\text { elderly }\end{array}$ & $\begin{array}{l}1.11(0.90-1.36) \\
p=0.3138\end{array}$ & $\begin{array}{l}1.23(0.96-1.56) \\
p=0.0873\end{array}$ & $\begin{array}{l}1.27(0.99-1.63) \\
p=0.0509\end{array}$ & $\begin{array}{l}1.21(0.95-1.55) \\
p=0.1121\end{array}$ \\
\hline \multicolumn{5}{|l|}{ CNS metastases } \\
\hline Yes vs no & $\begin{array}{l}1.17(0.91-1.51) \\
p=0.2127\end{array}$ & $\begin{array}{l}1.10(0.81-1.50) \\
\mathrm{p}=0.5355\end{array}$ & $\begin{array}{l}1.33(0.96-1.85) \\
p=0.0788\end{array}$ & $\begin{array}{l}1.31(0.95-1.81) \\
\mathrm{p}=0.1011\end{array}$ \\
\hline \multicolumn{5}{|l|}{ Bone metastases } \\
\hline Yes vs no & $\begin{array}{l}1.71(1.39-2.11) \\
p<0.0001\end{array}$ & $\begin{array}{l}1.80(1.41-2.30) \\
p<0.0001\end{array}$ & $\begin{array}{l}1.31(1.01-1.70) \\
p=0.0418\end{array}$ & $\begin{array}{l}1.27(0.98-1.66) ; \\
p=0.0684\end{array}$ \\
\hline
\end{tabular}

aHR, adjusted HR; BMI, body mass index; CNS, central nervous system; ECOG-PS, Easter Cooperative Oncology Group-Performance Status.

instead the adipose tissue as a functional reserve for patients receiving chemotherapy, a pronounced weight gain is related to an improved ORR, while a weight loss had a significant prognostic negative role for PFS and OS. In a post-hoc analysis from three phase III studies of patients receiving a platinum-based systemic therapy, Patel and colleagues evaluated the clinical outcomes according to a pre-planned weight gain cut-off (> vs $\leq 5 \%$ ), assessed by monitoring post-baseline weight variation till the maximum weight during treatment or at the 30-day post-study discontinuation follow-up visit. ${ }^{28}$ The authors reported that a weight gain of $>5 \%$ were significantly related to improved ORR, PFS and OS. ${ }^{28}$ However, the different methodology does not allow a direct comparison between our chemotherapy cohort and the cohort of Patel $e t a l .^{28}$

Our study has several limitations beyond the retrospective design and the consequent selection biases.
First, we cannot consider the chemotherapy cohort a proper validation cohort, because we did not perform any case-control matching; the percentage of elderly patients and patients with ECOG-PS $\geq 2$ are in fact significantly different. Considering the good clinical outcomes achieved in absolute terms, the chemotherapy cohort might had been positively biased. These findings are likely to be related to the clinicians attitude to treat with doublet chemotherapy more fit patients, compared with single agent pembrolizumab. Moreover, this positive selection might partially subtend to the lack of association between survival and BMI in the chemotherapy cohort. From this perspective, the analysis of the chemotherapy cohort collides with the evidences suggesting that also in NSCLC patients receiving chemotherapy a higher BMI is associated with an improved survival. ${ }^{11}$ Additional limitations include the data lack availability regarding comorbidities, the different sample size of the 


\section{OS; aHR for death (95\%Cl)}

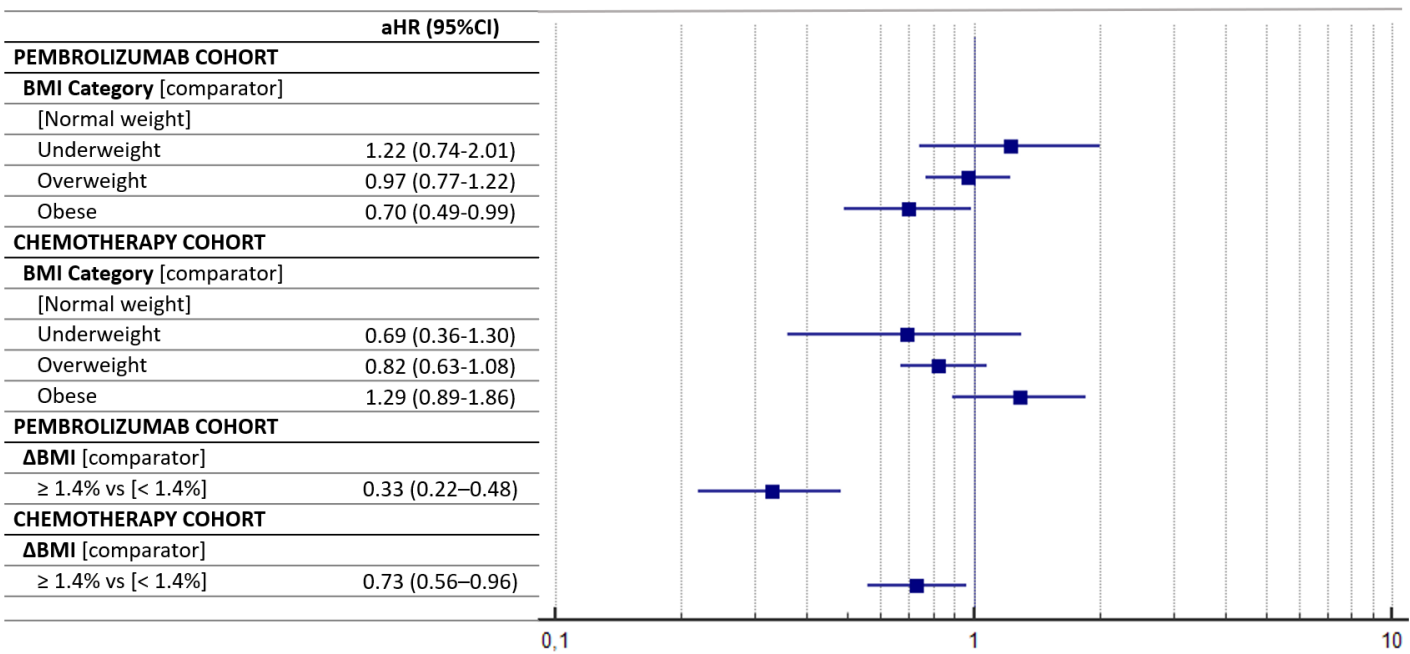

Figure 3 Forest plot graph for overall survival (OS). aHR, adjusted HR; BMI, body mass index.

two cohorts, as well as the median follow-up which was different. The chemotherapy cohort was not powered to detect statistically significant differences according to weight categories, moreover, being a historic cohort, we did not have data regarding PD-L1 expression for the subset of patients treated with chemotherapy. Nevertheless, considering the real-world prevalence of PD-L1 expression in NSCLC, we can assume that we one-third of the patients in the chemotherapy cohort had a PD-L1 expression of $\geq 50 \% .{ }^{29}$ Without having the weight data at pre-specified time points, we computed the $\Delta \mathrm{BMI}$ from treatment commencement to disease progression or to the last contact for censored patients, who, however, were progression free at the data cut-off and might had been positively selected with regards of the weight gain (due to better clinical condition). Conversely, progressed patients might have been negatively selected regarding the weight loss, even if the 4 weeks landmark selection was performed to mitigate this bias. In this respect, in our population, the probability of experience a BMI variation was conditional on the PFS, which was longer for the pembrolizumab cohort. Despite this, the median $\triangle \mathrm{BMI}$ was the same in the two cohort.

We have also to consider that with the weight-based dose overweight/obese patients have been exposed to higher dose intensity of pembrolizumab. Nevertheless, pharmacokinetic studies did not report definitive findings in exposure differences nor in safety profiles between the regimens (weight-based and fixed dose).$^{30}$ Furthermore, weight variation is inevitably related to the food intake, and chemotherapy is certainly associated to a higher incidence of anorexia, nausea and emesis compared with single agent pembrolizumab.

\section{CONCLUSION}

We confirmed that baseline obesity is associated to significantly improved ORR, PFS and OS also in metastatic
NSCLC patients with a PD-L1 expression of $\geq 50 \%$, receiving first line single agent pembrolizumab, but not among patients treated with chemotherapy. BMI variation during treatment is also significantly related to clinical outcomes, but it seems to have a different role for patients receiving first line pembrolizumab, compared with patients receiving first line platinum-based chemotherapy.

\section{Author affiliations}

${ }^{1}$ Department of Biotechnology and Applied Clinical Sciences, University of L'Aquila, L'Aquila, Italy

${ }^{2}$ Medical Oncology, St. Salvatore Hospital, L'Aquila, Italy

${ }^{3}$ Dana-Farber Cancer Institute, Harvard Medical School, Boston, Massachusetts, USA

${ }^{4}$ Medical Oncology, S. Orsola-Malpighi Hospital, University of Bologna, Bologna, Italy ${ }^{5}$ Medical Oncology, University Hospital of Parma, Parma, Emilia-Romagna, Italy

${ }^{6}$ Department of Medicine and Surgery, University of Parma, Parma, Italy

${ }^{7}$ Department of Translational Medicine and Surgery, Università Cattolica del Sacro

Cuore, Romae, Lazio, Italy

${ }^{8}$ Comprehensive Cancer Center, Fondazione Policlinico Universitario "A. Gemelli" IRCCS, Rome, Italy

${ }^{9}$ Oncology Department, Queen Alexandra University Hospital, Portsmouth Hospitals NHS Trust, Portsmuth, UK

${ }^{10}$ Department of Pulmonary Diseases, Erasmus Medical Center, Rotterdam, ZuidHolland, Netherlands

${ }^{11}$ Department of Oncology and Hematology, University Hospital Modena, Modena, Emilia-Romagna, Italy

${ }^{12}$ Medical Oncology Unit, Sant'Andrea Hospital, Roma, Lazio, Italy

${ }^{13}$ Medical Oncology, Azienda Ospedaliera San Gerardo, Monza, Lombardia, Italy

${ }^{14}$ Pneumo-Oncology Unit, San Camillo Forlanini Hospital, Roma, Lazio, Italy

${ }^{15}$ Thoracic Oncology Unit, Clinical Cancer Center IRCCS Istituto Tumori "Giovanni Paolo II", Bari, Puglia, Italy

${ }^{16}$ Department of Oncology, University of Turin, San Luigi Gonzaga University Hospital, Orbassano, Italy

${ }^{17}$ Oncology Clinic, Università Politecnica delle Marche, Ospedali Riuniti di Ancona, Ancona, Marche, Italy

${ }^{18}$ Thoracic Medical Oncology, Istituto Nazionale Tumori "Fondazione G Pascale" IRCCS, Napoli, Campania, Italy

${ }^{19}$ Lung Cancer Unit, IRCCS Ospedale Policlinnico San Martino, Genova, Liguria, Italy

${ }^{20}$ Medical Oncology, University Hospital Careggi, Firenze, Toscana, Italy

${ }^{21}$ Oncologia Medica e Terapia Biomolecolare, Azienda Ospedaliero-Universitaria

Ospedali Riuniti di Foggia, Foggia, Puglia, Italy 
${ }^{22}$ Department of Medical Oncology, Fondazione IRCCS Istituto Nazionale dei Tumori, Milano, Lombardia, Italy

${ }^{23}$ Medical Oncology Unit B, Policlinico Umberto I, Sapienza University of Rome,

Roma, Lazio, Italy

${ }^{24}$ Medical Oncology, ASUR District Area 4 Fermo, Ascoli Piceno, Italy

${ }^{25}$ Medical Oncology, Ospedali Riuniti Padova Sud "Madre Teresa di Calcutta",

Monselice, Veneto, Italy

${ }^{26}$ Pneumo-Oncology Unit, Ospedali dei Colli Monaldi Cotugno CTO, Napoli, Campania, Italy

${ }^{27}$ Oncology Unit, IRCCS Ospedale Sacro Cuore don Calabria, Negrar, Veneto, Italy

${ }^{28}$ Department of Medical, Oral and Biotechnological Sciences, University G.

D'Annunzio Chieti-Pescara, Chieti, $\mathrm{CH}$, Italy

${ }^{29}$ Clinical Oncology Unit, S.S. Annunziata Hospital, Chieti, Italy

${ }^{30}$ Medical Oncology, F. Spaziani Hospital, Frosinone, Lazio, Italy

${ }^{31}$ Medical Oncology, Ospedale Santa Maria Goretti, Latina, Lazio, Italy

${ }^{32}$ Medical Oncology Unit, Fondazione IRCCS Ca' Granda Ospedale Maggiore di Milano Policlinico, Milano, Lombardia, Italy

${ }^{33}$ Medical Oncology, Campus Bio-Medico University, Rome, Italy

${ }^{34}$ Department of Oncology, University Hospital Santa Maria della Misericordia, Udine, Friuli-Venezia Giulia, Italy

${ }^{35}$ Medical Oncology, ASST-Sette Laghi, Varese, Lombardia, Italy

${ }^{36}$ Department of Oncology and Hematology, AUSL della Romagna, Ravenna, Emilia-

Romagna, Italy

${ }^{37}$ Oncology Department, University Hospital of Geneva, Geneve, Genève, Switzerland

${ }^{38}$ Oncology Unit, ASST Bergamo Ovest, Treviglio, Lombardia, Italy

Twitter Emilio Bria @emilio.bria and Giuseppe L Banna @gbanna74

Acknowledgements A special thank to the 'Consorzio Interuniversitario Nazionale per la Bio-Oncologia' for their support in this study.

Contributors All authors contributed to the publication according to the ICMJE guidelines for the authorship (study conception and design, acquisition of data, analysis and interpretation of data, drafting of manuscript, critical revision). All authors read and approved the submitted version of the manuscript (and any substantially modified version that involves the author's contribution to the study). Each author has agreed both to be personally accountable for the author's own contributions and to ensure that questions related to the accuracy or integrity of any part of the work, even ones in which the author was not personally involved, are appropriately investigated, resolved, and the resolution documented in the literature.

Funding The authors have not declared a specific grant for this research from any funding agency in the public, commercial or not-for-profit sectors.

Competing interests $\mathrm{AC}$ received speaker fees and grant consultancies by Astrazeneca, MSD, BMS, Roche, Novartis and Astellas. JA reports receiving commercial research grants from Amphera and Roche, holds ownership interest (including patents) in Amphera BV, and is a consultant/advisory board member for Amphera, Boehringer Ingelheim, Bristol-Myers Squibb, Eli-Lilly, MSD and Roche. EB received speakers' and travels' fee from MSD, Astra-Zeneca, Celgene, Pfizer, Helsinn, Eli-Lilly, BMS, Novartis and Roche. EB received consultant's fee from Roche, Pfizer. EB received institutional research grants from Astra-Zeneca, Roche. MT received speaker fees and grant consultancies by Astrazeneca, Pfizer, Eli-Lilly, BMS, Novartis, Roche, MSD, Boehringer Ingelheim, Otsuka, Takeda and Pierre Fabre. AM received speaker fees by Astra, Roche, BMS, MSD, Boehringer, Pfizer, Takeda. FM received grant consultancies by MSD and Takeda. RG received speaker fees and grant consultancies by Astrazeneca and Roche. FP received grant consultancies by MSD and Astrazeneca. AF received grant consultancies by Roche, Pfizer, Astellas and BMS. AA received grant consultancies by Takeda, MSD, BMJ, Astrazeneca, Roche and Pfizer. RC received speaker fees by BMS, MSD, Takeda, Pfizer, Roche and Astrazeneca. CG received speaker fees/grant consultancies by Astrazeneca, BMS, Boehringer-Ingelheim, Roche and MSD. MR received honoraria for scientific events by Roche, Astrazeneca, Bristol-Myers Squibb, Merck Sharp \& Dohme and Boehringer Ingelheim.

\section{Patient consent for publication Not required.}

Ethics approval All patients provided written, informed consent to treatment with immunotherapy. The procedures followed were in accordance with the precepts of good clinical practice and the declaration of Helsinki. The study was approved by the respective local ethical committees on human experimentation of each institution, after previous approval by the coordinating center (Comitato Etico per le provice di L'Aquila e Teramo, verbale N.15 del 28 Novembre 2019).
Provenance and peer review Not commissioned; externally peer reviewed.

Data availability statement The datasets used during the present study are available from the corresponding author upon reasonable request.

Open access This is an open access article distributed in accordance with the Creative Commons Attribution Non Commercial (CC BY-NC 4.0) license, which permits others to distribute, remix, adapt, build upon this work non-commercially, and license their derivative works on different terms, provided the original work is properly cited, appropriate credit is given, any changes made indicated, and the use is non-commercial. See http://creativecommons.org/licenses/by-nc/4.0/.

\section{ORCID iDs}

Alessio Cortellini http://orcid.org/0000-0002-1209-5735

Alessandro Morabito http://orcid.org/0000-0002-1319-9608

\section{REFERENCES}

1 Cortellini A, Bersanelli M, Buti S, et al. A multicenter study of body mass index in cancer patients treated with anti-PD-1/PD-L1 immune checkpoint inhibitors: when overweight becomes favorable. $J$ Immunother Cancer 2019;7:57.

2 McQuade JL, Daniel CR, Hess KR, et al. Association of bodymass index and outcomes in patients with metastatic melanoma treated with targeted therapy, immunotherapy, or chemotherapy: a retrospective, multicohort analysis. Lancet Oncol 2018;19:310-22.

3 De Giorgi U, Procopio G, Giannarelli D, et al. Association of systemic inflammation index and body mass index with survival in patients with renal cell cancer treated with nivolumab. Clin Cancer Res 2019;25:3839-46.

4 Martini DJ, Kline MR, Liu Y, et al. Adiposity may predict survival in patients with advanced stage cancer treated with immunotherapy in phase 1 clinical trials. Cancer 2020;126:575-82.

5 Cortellini A, Bersanelli M, Santini D, et al. Another side of the association between body mass index (BMI) and clinical outcomes of cancer patients receiving programmed cell death protein-1 (PD-1)/ Programmed cell death-ligand 1 (PD-L1) checkpoint inhibitors: A multicentre analysis of immune-related adverse events. Eur J Cancer 2020;128:17-26.

6 Ichihara E, Harada D, Inoue K, et al. The impact of body mass index on the efficacy of anti-PD-1/PD-L1 antibodies in patients with nonsmall cell lung cancer. Lung Cancer 2020;139:140-5.

7 Popinat G, Cousse S, Goldfarb L, et al. Sub-cutaneous fat mass measured on multislice computed tomography of pretreatment PET/ $\mathrm{CT}$ is a prognostic factor of stage IV non-small cell lung cancer treated by nivolumab. Oncoimmunology 2019;8:e1580128.

8 Kichenadasse G, Miners JO, Mangoni AA, et al. Association between body mass index and overall survival with immune checkpoint inhibitor therapy for advanced non-small cell lung cancer. JAMA Oncol 2020;6:512-8.

9 Wang Z, Aguilar EG, Luna JI, et al. Paradoxical effects of obesity on $T$ cell function during tumor progression and PD-1 checkpoint blockade. Nat Med 2019;25:141-51.

10 Mullen M, Gonzalez-Perez RR. Leptin-Induced JAK/STAT signaling and cancer growth. Vaccines 2016;4:26.

11 Petrelli F, Cortellini A, Indini A, et al. Obesity paradox in patients with cancer: a systematic review and meta-analysis of $6,320,365$ patients. medRxiv 2020.

12 Cortellini A, Tiseo M, Banna GL, et al. Clinicopathologic correlates of first-line pembrolizumab effectiveness in patients with advanced NSCLC and a PD-L1 expression of $\geq 50 \%$. Cancer Immunol Immunother 2020;375.

13 Cortellini A, Friedlaender A, Banna GL, et al. Immune-related Adverse Events of Pembrolizumab in a Large Real-world Cohort of Patients With NSCLC With a PD-L1 Expression $\geq 50 \%$ and Their Relationship With Clinical Outcomes. Clin Lung Cancer 2020. doi:10.1016/j. cllc.2020.06.010. [Epub ahead of print: 21 Jun 2020].

14 Eisenhauer EA, Therasse P, Bogaerts J, et al. New response evaluation criteria in solid tumours: revised RECIST guideline (version 1.1). Eur J Cancer 2009;45:228-47.

15 Woolley KK. How variables uncorrelated with the dependent variable can actually make excellent predictors: the important suppressor variable case. Southwest Educational Research Association Annual Meeting proceedings, 1997.

16 Thompson FT, Levine DU. Examples of easily Explainable suppressor variables in multiple regression research. Multiple Linear Regression Viewpoints 1997;24:11-13.

17 "Stopping stepwise: Why stepwise selection is bad and what you should use instead". Available: https://towardsdatascience.com/ 
stopping-stepwise-why-stepwise-selection-is-bad-and-what-youshould-use-instead-90818b3f52df [Accessed 29 Mar 2020].

18 Gridelli C, Balducci L, Ciardiello F, et al. Treatment of elderly patients with non-small-cell lung cancer: results of an international expert panel meeting of the Italian association of thoracic oncology. Clin Lung Cancer 2015;16:325-33.

19 Bleeker SE, Moll HA, Steyerberg EW, et al. External validation is necessary in prediction research: a clinical example. J Clin Epidemiol 2003;56:826-32.

20 Gelibter A, Occhipinti M, Pisegna S, et al. Status of correlation between $\mathrm{BMI}$ and response to immunocheck-point inhibitor in advanced non-small-cell lung cancer. Lung Cancer Manag 2020;9:LMT26.

21 Abdel-Rahman O. Pre-Diagnostic body mass index trajectory in relationship to lung cancer incidence and mortality; findings from the PLCO trial. Expert Rev Respir Med 2019;13:1029-35.

22 Aldrich MC, Grogan EL, Munro HM, et al. Stage-adjusted lung cancer survival does not differ between low-income blacks and whites. J Thorac Oncol 2013;8:1248-54.

23 Nonemaker JM, Garrett-Mayer E, Carpenter MJ, et al. The risk of dying from lung cancer by race: a prospective cohort study in a biracial cohort in Charleston, South Carolina. Ann Epidemiol 2009;19:304-10.

24 Qi Y, Schild SE, Mandrekar SJ, et al. Pretreatment quality of life is an independent prognostic factor for overall survival in patients with advanced stage non-small cell lung cancer. $J$ Thorac Oncol 2009;4:1075-82.

25 Argilés JM, Busquets S, Stemmler B, et al. Cancer cachexia: understanding the molecular basis. Nat Rev Cancer 2014;14:754-62.

26 Scott HR, McMillan DC, Forrest LM, et al. The systemic inflammatory response, weight loss, performance status and survival in patients with inoperable non-small cell lung cancer. Br J Cancer 2002;87:264-7.

27 Dahlberg SE, Schiller JH, Bonomi PB, et al. Body mass index and its association with clinical outcomes for advanced non-small-cell lung cancer patients enrolled on eastern cooperative Oncology group clinical trials. J Thorac Oncol 2013;8:1121-7.

28 Patel JD, Pereira JR, Chen J, et al. Relationship between efficacy outcomes and weight gain during treatment of advanced, nonsquamous, non-small-cell lung cancer patients. Ann Oncol 2016;27:1612-9.

29 Dietel M, Savelov N, Salanova R, et al. Real-World prevalence of programmed death ligand 1 expression in locally advanced or metastatic non-small-cell lung cancer: the global, multicenter EXPRESS study. Lung Cancer 2019;134:174-9.

30 Centanni M, Moes DJAR, Trocóniz IF, et al. Clinical pharmacokinetics and pharmacodynamics of immune checkpoint inhibitors. Clin Pharmacokinet 2019;58:835-57. 